\title{
Diagnóstico microbiológico y vigilancia epidemiológica de la campilobacteriosis en Chile: Situación actual y desafíos futuros
}

\author{
Microbial diagnosis and epidemiological surveillance of campylobacteriosis \\ in Chile: Present state and further challenges
}

\author{
Luis Collado ${ }^{1,2}$
}

'Instituto de Bioquímica y Microbiología, Facultad de Ciencias, Universidad Austral de Chile, Campus Isla Teja, Valdivia, Chile.

${ }^{2}$ Núcleo Milenio Biología de la Microbiota Intestinal, Santiago, Chile.

El autor declara no tener conflictos de interés en este tema.

Este trabajo fue financiado por Fondo Nacional de Ciencia y Tecnología de Chile (FONDECYT 1200125).

Además, el autor agradece el soporte del Núcleo Milenio Biología de la Microbiota Intestinal (NU-GUTmicro).

Recibido: 9 de diciembre de 2019 / Aceptado: 2 de abril de 2020

\section{Resumen}

Campylobacter spp. es considerado el primer agente etiológico de diarrea en países desarrollados, y el segundo o tercero en países en vías de desarrollo. La elevada incidencia de gastroenteritis por Campylobacter spp. y sus posibles secuelas post-infección (artritis reactiva, el síndrome de Miller-Fisher o el síndrome de GuillainBarré) le confieren gran importancia desde el punto de vista socioeconómico. Sin embargo, en la mayoría de los países en vías de desarrollo no existe diagnóstico microbiológico rutinario de este patógeno. En Chile, la campilobacteriosis es notificable desde 1983. Sin embargo, la falta de diagnóstico rutinario por parte de los laboratorios clínicos ha dificultado conocer la verdadera prevalencia de este patógeno, tanto en infecciones intestinales como extra-intestinales. Además, a pesar que la campilobacteriosis es una enfermedad zoonótica, el diagnóstico de este patógeno no está considerado dentro del Reglamento Sanitario de los Alimentos de Chile. Todo esto se traduce en una falta de conocimiento sobre la epidemiología de la enfermedad por Campylobacter spp en Chile. Por lo tanto, es objetivo de esta revisión analizar la situación de la campilobacteriosis en las últimas dos décadas en Chile y determinar cuáles son los desafíos que quedan para lograr un diagnóstico y vigilancia efectivos en el país.

Palabras clave: Campylobacter; diagnóstico; resistencia antimicrobiana; epidemiología; Chile.

\begin{abstract}
Campylobacter spp. is considered as the first etiologic agent of diarrhea in developed countries and the second or third in developing countries. The high incidence of Campylobacter gastroenteritis and its possible post-infection sequelae (reactive arthritis, Miller-Fisher syndrome or Guillain-Barré syndrome) give it great importance from the socioeconomic point of view. However, in most developing countries there is no routine microbial diagnosis of this pathogen. In Chile, campylobacteriosis is notifiable since 1983. However, the lack of routine diagnosis by clinical laboratories has made it difficult to know the true prevalence of this pathogen in both intestinal and extra-intestinal infections. In addition, although campylobacteriosis is a zoonotic disease, the diagnosis of this pathogen is not considered within the Chilean Food Sanitary Regulations. All this results in a lack of knowledge about the epidemiology of Campylobacter spp disease in Chile. Therefore, the objective of this review is to analyze the situation of campylobacteriosis in the last two decades in Chile and to determine the challenges that remain to achieve an effective microbial diagnosis and epidemiologic surveillance.
\end{abstract}

Keywords: Campylobacter; diagnosis; antibiotic resistance; epidemiology; Chile. 
temente (febrero de 2017), la OMS publicó una lista de 12 bacterias con alta resistencia a antimicrobianos donde se incluye a Campylobacter como un patógeno de alta prioridad debido a su alta resistencia a fluoroquinolonas universalmente ${ }^{8}$.

En esta revisión, se pretende analizar la situación del diagnóstico microbiológico, resistencia antimicrobiana y vigilancia epidemiológica de la campilobacteriosis en base a los antecedentes bibliográficos generados en Chile durante las últimas dos décadas, conocer la situación actual, los avances efectuados en este período y evaluar desafíos futuros. su principal vía de transmisión, por lo que es considerado un patógeno zoonótico ${ }^{2}$.

Las especies prevalentes en casos de gastroenteritis son Campylobacter jejuni y Campylobacter coli, las que representan aproximadamente 80 a $90 \%$ y 4 a $10 \%$, respectivamente, de todas las cepas aisladas en laboratorios clínicos $^{1,2}$. Los cuadros clínicos más comunes producidos por estas especies son diarrea aguda (frecuentemente con presencia de sangre en las heces), dolor abdominal, fiebre, cefalea, náuseas y/o vómitos, y duran por lo general de tres a seis días ${ }^{1}$. Con menor frecuencia, también se ha observado que estas bacterias pueden producir otras complicaciones como bacteriemia, hepatitis, pancreatitis y abortos ${ }^{1}$. Además, entre las complicaciones o secuelas posteriores a la infección figuran la artritis reactiva (que puede durar varios meses) y trastornos neurológicos como el síndrome de Miller-Fisher o el síndrome de GuillainBarré (SGB) ${ }^{3}$. Estas complicaciones neurológicas son enfermedades graves; en el caso del SGB suelen requerir cuidados intensivos en aproximadamente $20 \%$ de los casos y su tasa de mortalidad en los países industrializados está entre 3 y $10 \%{ }^{4}$.

Uno de los principales problemas para comprender la importancia de la campilobacteriosis a nivel global es la falta de diagnóstico rutinario en la mayoría de los países menos desarrollados. En el año 2000, la Organización Mundial de la Salud (OMS) recomendó que, en los países en vías de desarrollo, todas las muestras fecales analizadas para Salmonella y Shigella, también deberían ser analizadas para Campylobacter spp., ${ }^{4,5}$. Lamentablemente, esta recomendación no ha sido fácil de implementar debido principalmente a una serie de razones económicas. De hecho, revisiones recientes sobre la epidemiología global de Campylobacter spp. ratifican que en la mayoría de estos países no existe diagnóstico rutinario de este patógeno $\mathrm{y}$, por lo tanto, tampoco una vigilancia epidemiológica efectiva ${ }^{1,5}$.

La falta de diagnóstico de Campylobacter ha tenido un efecto directo en la deficiente vigilancia de su resistencia antimicrobiana en países en vías de desarrollo. Lo cual se agrava por el aumento progresivo de la resistencia a distintos tipos de antimicrobianos en las últimas décadas ${ }^{6}$ y por el surgimiento de cepas multi-resistentes ${ }^{7}$. Recien-

\section{Diagnóstico microbiológico y prevalencia de Campylobacter spp.}

En Chile, la mayoría de los laboratorios clínicos no tienen implementado el diagnóstico microbiológico de Campylobacter sp de forma rutinaria ${ }^{9,10}$. El principal obstáculo en la implementación de este diagnóstico son los altos costos de los medios de cultivo selectivos y los equipos y/o materiales necesarios para generar una atmósfera micro-aeróbica estricta ${ }^{11,12}$. Existen varios medios selectivos en base a agar suplementado con sangre de cordero (Agar Skirrow, Agar Preston, Agar Bolton, entre otros) o carbón activado (agar CCDA), a los que generalmente se les agrega una mezcla antimicrobiana incluyendo algún $\beta$-lactámico como cefoperazona, ya que Campylobacter es intrínsecamente resistente a éstos ${ }^{4}$. La condición de micro-aerobiosis (5\% de oxígeno, $10 \%$ de dióxido de carbono y $85 \%$ de nitrógeno), generalmente se obtiene utilizando sobres comerciales desechables (CampyGen de Oxoid ${ }^{\circledR}$ o GasPak EZ Campy Sachet ${ }^{\circledR}$ de Becton Dickinson, entre otros) que se disponen dentro de jarras anaeróbicas o, alternativamente, se puede realizar un método de evacuación y reemplazo de gases en las jarras anaerobias ${ }^{13}$. Por otra parte, los métodos de identificación fenotípicos no tienen mucha utilidad para la caracterización de Campylobacter a nivel de especie, debido a su baja actividad bioquímica. De hecho, sólo la especie $C$. jejuni es identificada correctamente mediante pruebas bioquímicas, ya que es la única campilobacteria de importancia clínica que hidroliza el hipurato ${ }^{13}$. Por lo que, en los escasos laboratorios donde se han implementado los métodos de aislamiento, generalmente la identificación se realiza sólo a nivel de género.

Debido a estos problemas técnicos y económicos, en Chile se ha recomendado diagnosticar la campilobacteriosis mediante la observación microscópica directa de las muestras fecales utilizando la tinción violeta-bicarbonato (VB) o también llamada tinción de Hucker ${ }^{11}$. Sin embargo, este método no reemplaza el cultivo, ya que sólo permite un diagnóstico presuntivo, no es posible identificar las 


\begin{tabular}{|c|c|c|c|c|c|}
\hline \multirow[t]{2}{*}{ Ciudad } & \multirow[t]{2}{*}{ Métodos de aislamiento y/o detección } & \multicolumn{2}{|c|}{ Muestras fecales } & \multirow[t]{2}{*}{ Especies ( $n$ ) } & \multirow[t]{2}{*}{ Referencias } \\
\hline & & $\mathbf{n}$ & $\%$ & & \\
\hline \multirow[t]{2}{*}{ Santiago } & Cultivo (agar Skirrow) & 164 & 9,8 & C. jejuni (16) & 12 \\
\hline & Observación microscópica (tinción de Hucker) & 164 & 3,7 & Campylobacter sp. (6) & \\
\hline \multirow[t]{2}{*}{ Santiago } & Cultivo (agar CCDA) & 66 & 15,2 & Campylobacter sp. (10) & 35 \\
\hline & Inmunocromatografía (Immuno Card Campy STAT!@) & 66 & 15,2 & Campylobacter sp. (10) & \\
\hline \multirow[t]{3}{*}{ Santiago } & Cultivo (agar Campylosel) & 60 & 18 & $\begin{array}{l}\text { C. jejuni (10) } \\
\text { Campylobacter sp. (1) }\end{array}$ & 36 \\
\hline & Inmunocromatografía (CerTest Campylobacter ${ }^{\circledR}$ ) & 60 & 15 & Campylobacter sp. (9) & \\
\hline & Inmunocromatografía (Immuno Card Campy STAT!®) & 60 & 18 & Campylobacter sp. (11) & \\
\hline \multirow[t]{2}{*}{ Santiago } & Cultivo (agar Campylosel) & 750 & 6,1 & $\begin{array}{l}\text { C. jejuni (41) } \\
\text { C. coli (4) }\end{array}$ & 15 \\
\hline & Observación microscópica (tinción de Hucker) & 750 & 0,4 & Campylobacter sp. (3) & \\
\hline Santiago & FilmArray GI Panel & 199 & 12,7 & Campylobacter sp. & 17 \\
\hline Valparaíso & Cultivo (agar CASA) & 350 & 8 & $\begin{array}{l}\text { C. jejuni (26) } \\
\text { C. coli (2) }\end{array}$ & 19 \\
\hline Concepción & Cultivo (agar Skirrow) & 291 & 2,1 & C. jejuni (6) & 31 \\
\hline \multirow[t]{2}{*}{ Valdivia } & Cultivo (agar mCCDA y filtración sobre agar sangre) & 140 & 10 & C. jejuni (14) & 16 \\
\hline & Detección por RPC & 140 & 26 & $\begin{array}{l}\text { Campylobacter sp. (26) } \\
\text { C. jejuni (15) } \\
\text { C. concisus (16) } \\
\text { C. ureolyticus (5) }\end{array}$ & \\
\hline
\end{tabular}

especies, determinar la sensibilidad antimicrobiana ni tipificar las cepas asociadas a la infección. A pesar del escaso diagnóstico microbiológico en el medio nacional, en el período 2015-2016 se notificaron tres brotes de enfermedades trasmitidas por alimentos (ETA) atribuibles a Campylobacter spp. ${ }^{14}$.

Para conocer la prevalencia de Campylobacter spp. en Chile, se ha revisado los datos generados por grupos de investigación en las últimas dos décadas (Tabla 1). A pesar de que los estudios no son muy comparables, debido a la gran diversidad de métodos diagnósticos utilizados (observación microscópica, cultivo en medios selectivos, inmunocromatografía y métodos moleculares), se puede observar que la prevalencia de Campylobacter spp. mediante cultivo está en el rango entre 2,1 y $18 \%$. La mayor parte de estos estudios se han desarrollado en la Región Metropolitana, siendo escasos los reportes en otras regiones o zonas del país. En el estudio de Porte y cols., ${ }^{15}$ se demostró que, mediante cultivo, Campylobacter tiene una prevalencia muy similar a Salmonella $(6,1$ y
$6,5 \%$, respectivamente). Este trabajo también demostró la falta de sensibilidad del diagnóstico microscópico mediante tinción $\mathrm{VB}$ en comparación con el cultivo de Campylobacter ( 0,4 y $6,1 \%$, respectivamente). Sin embargo, otros autores reportan mayores valores de sensibilidad para el método microscópico directo ${ }^{12}$, probablemente debido a que éste es un método operadordependiente. La alta sensibilidad del cultivo ha sido ratificada al compararla con detección molecular. De hecho, en el estudio de Collado y cols. ${ }^{16}$, se detectó $C$. jejuni en proporciones muy similares mediante cultivo y pesquisa mediante reacción de polimerasa en cadena (RPC), 10 y 10,7\%, respectivamente.

Recientemente, se utilizó el método FilmArray GI Panel® (BioMérieux) para la detección directa de enteropatógenos bacterianos, virales y parasitarios en muestras fecales de pacientes adultos en un servicio de urgencias en Santiago y se demostró que Campylobacter spp., con una prevalencia de $12,7 \%$, fue el segundo patógeno más común después de Escherichia coli en- 
está claro en nuestro país cuál es la contribución relativa de cada una de las fuentes de origen animal a la campilobacteriosis humana. casos de campilobacteriosis hubo co-infección con algún otro enteropatógeno. Lamentablemente, este método diagnóstico no se ha implementado de forma masiva en Chile y además presenta un costo elevado lo que impide su uso rutinario.

A pesar de los escasos datos sobre la prevalencia de Campylobacter spp., existen algunas tendencias importantes en relación a su epidemiología en nuestro territorio. Por ejemplo, los datos más antiguos que existen en Chile generalmente atribuían la campilobacteriosis casi exclusivamente a pacientes en edad pediátrica ${ }^{18}$. Sin embargo, estudios más recientes han demostrado que este agente es detectado prácticamente en pacientes de todas las edades con predominancia en los grupos etarios de 1 a 9 años $y$ de 10 a 19 años ${ }^{10,12,15,16,19}$, de manera parecida a lo que ocurre en países industrializados donde la mayoría de los casos se dan en niños y adultos jóvenes. Esto podría tener relación con un cambio en la epidemiología de la campilobacteriosis a medida que han ido mejorando las medidas sanitarias nacionales.

En Chile, al igual que en el resto del mundo, la especie prevalente en infecciones entéricas es $C$. jejuni seguida de $C$. coli ${ }^{10,15}$. Por otra parte, la especie más común en infecciones sistémicas es Campylobacter fetus ${ }^{10}$. Además de las especies mencionadas, existen varias campilobacterias emergentes, reportadas ocasionalmente, tanto en infecciones intestinales como extra-intestinales, tales como Campylobacter upsaliensis, Campylobacter lari ${ }^{10}$, Campylobacter concisus, Arcobacter butzleri ${ }^{16}$ y Helicobacter entero-hepáticos ${ }^{20}$. Sin embargo, el rol clínico de estas especies no es bien comprendido en la actualidad y probablemente seguirán siendo sub-diagnosticadas y subestimadas hasta que contemos con métodos diagnósticos más precisos.

La falta de diagnóstico de Campylobacter no sólo se limita a los laboratorios clínicos. De hecho, en el caso de alimentos, este patógeno no se encuentra incluido en el Reglamento Sanitario de los Alimentos (RSA) del Ministerio de Salud de Chile ${ }^{21}$ y los datos de la presencia de especies de Campylobacter spp. en plantas de procesamiento chilenas son limitados ${ }^{14}$. Sin embargo, algunos estudios desarrollados en el centro y sur de Chile han demostrado que $C$. jejuni y $C$. coli presentan una alta prevalencia en muestras fecales de animales de granja y en la carne derivada de éstos ${ }^{22,23}$. De hecho, recientemente el Programa Nacional de Vigilancia Microbiológica en Alimentos del Ministerio de Salud, informó que, de un total de 365 muestras de carne de ave cruda tomadas a lo largo del país, $44,7 \%$ fueron positivas para Campylobacter spp. $^{24}$. En Chile, $C$. jejuni y C. coli también han sido detectadas en muestras fecales de animales de compañía $\mathrm{y}$ en animales silvestres ${ }^{25-27}$. Aunque, por el momento no

\section{Tratamiento de la campilobacteriosis y resistencia antimicrobiana}

La campilobacteriosis humana es, por lo general, una enfermedad aguda y auto-limitada. En la mayoría de los casos, la reposición de fluidos y electrolitos es el único tratamiento recomendado. Sin embargo, la terapia antimicrobiana es necesaria en casos graves y prolongados ${ }^{6}$. Los antimicrobianos más utilizados para el tratamiento de la campilobacteriosis son los macrólidos -eritromicina- o azálidas -azitromicina- (tratamiento de elección) y fluoroquinolonas -ciprofloxacina- (tratamiento de segunda línea) ${ }^{23}$. Sin embargo, en las últimas décadas, la resistencia de Campylobacter sp. a éstos y otras familias de antimicrobianos (p. ej.: tetraciclinas o aminoglucósidos usados en infecciones sistémicas) se ha reportado globalmente ${ }^{6}$. Debido a esto, la OMS recomienda realizar pruebas de susceptibilidad antimicrobiana, tanto para proporcionar una orientación sobre el tratamiento adecuado de la campilobacteriosis, como para contribuir al conocimiento epidemiológico y a la vigilancia de la resistencia en Campylobacter spp. ${ }^{4}$. Sin embargo, en la práctica, el análisis de susceptibilidad es raramente realizado y generalmente se prescribe un tratamiento empírico?.

Los datos sobre resistencia antimicrobiana de cepas clínicas de Campylobacter en Chile durante los últimos 20 años están resumidos en la Tabla 2. Al igual que en los métodos de diagnóstico, las técnicas utilizadas para determinar resistencia son muy variadas (epsilometría o Etest ${ }^{\circledR}$, difusión en agar, dilución en caldo o agar) así como los puntos de corte utilizados (CLSI, EUCAST, NARMS, entre otros). Además, los datos se concentran en estudios desarrollados en la zona central (Santiago-Valparaíso) o sur de Chile (Concepción-Valdivia), no existiendo datos disponibles sobre la situación en el norte del país.

En la mayoría de los estudios sólo se han analizado eritromicina y ciprofloxacina y, al igual que en los reportes internacionales, la resistencia a ciprofloxacina es alarmante ${ }^{8}$. En Valdivia se ha evidenciado el cambio más notorio en el patrón de resistencia a nivel nacional. De hecho, se pasó de una completa sensibilidad a ciprofloxacina en cepas clínicas de C. jejuni aisladas entre los años 1997-1998 ${ }^{28}$, a casi la mitad de las cepas resistentes a este antimicrobiano, dos décadas más tarde ${ }^{29}$. $\mathrm{El}$ aumento de la resistencia a tetraciclina también es considerable; en el año 2000 la resistencia alcanzaba a $1,8 \%$ de las cepas y los datos más actuales la sitúan sobre $20 \%$ (Tabla 2). No se conoce la razón exacta del aumento de resistencia a estos tipos de antimicrobianos, aunque se ha sugerido que puede 


\section{—}

\begin{tabular}{|c|c|c|c|c|c|c|c|c|c|c|}
\hline \multirow[t]{2}{*}{ Ciudad } & \multirow{2}{*}{$\begin{array}{l}\mathrm{n} \text { de } \\
\text { cepas }\end{array}$} & \multirow[t]{2}{*}{ Especie } & \multirow[t]{2}{*}{ Metodología } & \multicolumn{6}{|c|}{ Porcentaje (\%) de cepas resistentes } & \multirow[t]{2}{*}{ Referencias } \\
\hline & & & & CIP & ERI & AZI & TET & GEN & AMP & \\
\hline Santiago & 73 & C. jejuni & Epsilometría $\left(\right.$ E-test $\left.{ }^{\circledR}\right)$ & 32,4 & 0 & 0 & NP & NP & 1,4 & 9 \\
\hline Santiago & 55 & C. jejuni & Microdilución en caldo & 60 & 0 & NP & NP & NP & NP & 37 \\
\hline \multirow[t]{2}{*}{ Santiago } & 66 & C. jejuni & Dilución en agar & 30,3 & 1,5 & NP & 24,3 & 0 & NP & 23 \\
\hline & 7 & C. coli & & 57,2 & 28,5 & NP & 28,5 & 0 & NP & \\
\hline Santiago & 17 & C. jejuni & Difusión en agar/dilución en agar & 52,9 & 5,9 & NP & $23,5^{*}$ & $0^{*}$ & NP & $\begin{array}{l}29 \\
33\end{array}$ \\
\hline Santiago & 14 & Campylobacter sp. & Epsilometría $\left(\mathrm{E}\right.$-test $\left.{ }^{\circledR}\right)$ & 35,7 & 7,1 & NP & 21,4 & NP & NP & 15 \\
\hline Valparaíso & 28 & Campylobacter sp. & Difusión en agar & 63 & 3,7 & NP & 25,9 & 0 & 71.4 & 19 \\
\hline Concepción & 6 & C. jejuni & Difusión en agar/ dilución en agar & 33,3 & 0 & NP & NP & NP & NP & 31 \\
\hline Valdivia & 108 & C. jejuni & Epsilometría (E-test $₫) /$ dilución en agar & 0 & 0 & NP & 1,8 & 0 & 6,5 & 28 \\
\hline Valdivia & 33 & C. jejuni & Difusión en agar/dilución en agar & 45,5 & 0 & NP & $17,4^{*}$ & $0^{*}$ & NP & $\begin{array}{l}29 \\
33\end{array}$ \\
\hline
\end{tabular}

estar asociado al uso veterinario de antimicrobianos ${ }^{30}$. Es sabido que la cantidad de quinolonas y tetraciclinas destinadas para el uso veterinario en Chile es muy superior al usado en medicina humana ${ }^{30,31}$. Además, el hecho de que gran parte de los aislados resistentes a ciprofloxacina provengan de niños, en quienes no se recomienda usar este tipo de antimicrobiano como tratamiento empírico de diarrea, hace suponer que la infección ha sido provocada por bacterias previamente resistentes ${ }^{9,32}$.

Por otra parte, en los últimos años también se ha comenzado a notar una incipiente resistencia a eritromicina $^{15,23,29}$. Hasta el momento, no se ha detectado resistencia a gentamicina en cepas clínicas aisladas en Chile (Tabla 2) y, por lo tanto, este antimicrobiano se convierte en un buen recurso terapéutico para infecciones sistémicas.

Los mecanismos moleculares responsables de la resistencia han sido evaluados recientemente en un grupo de cepas clínicas aisladas en Santiago y Valdivia. Se demostró que la mutación C257T de la "región determinante de la resistencia a quinolonas" (RDRQ) del gen gyrA (ADN girasa o topoisomerasa II), así como la mutación $\mathrm{A} 2075 \mathrm{G}$ del gen que codifica para el gen $23 \mathrm{~S}$ ARNr y la presencia del gen tet(o) serían los principales determinantes moleculares responsable de la resistencia a ciprofloxacina, eritromicina y tetraciclina, respectiva- mente, en cepas aisladas en Chile, además de la presencia de la bomba de expulsión $\mathrm{CmeABC}^{33}$.

En esta revisión, no se ha incluido los datos de resistencia encontrada en cepas de Campylobacter aislados de alimentos en Chile. Sin embargo, esto fueron recientemente revisados por Lapierre y cols. ${ }^{23}$, donde destaca la alta resistencia a fluoroquinolonas, tetraciclinas y macrólidos en cepas aisladas de carne de pollo y vacuno, soportando así, la teoría de que el origen del aumento de la resistencia estaría influenciada por el uso de antimicrobianos en el área veterinaria.

\section{Vigilancia epidemiológica}

La notificación y derivación de aislados de Campylobacter spp. al laboratorio de referencia del Instituto de Salud Pública (ISP) de Chile es baja, debido principalmente a que la mayoría de los laboratorios clínicos no estudian este agente por la ya mencionada falta de implementación de técnicas de diagnóstico ${ }^{31}$. Esto, a pesar que desde el año 1983, Campylobacter es clasificado como un agente de vigilancia de laboratorio según lo establece el reglamento sobre notificación de enfermedades transmisibles de declaración obligatoria DS $\mathrm{N}^{\circ} 158^{10}$. Esta precaria 
principales o también llamados complejos clonales (CC). Esto también sugiere que probablemente existe una diseminación clonal de cepas resistentes a este u otro grupo de antimicrobianos en Chile ${ }^{29}$. Lamentablemente, no existen más datos de cepas chilenas disponibles en la base de datos PubMLST (https://pubmlst.org/campylobacter/) (revisado por última vez el 02 de diciembre de 2019), lo que impide comprender de mejor manera la epidemiología de la campilobacteriosis en nuestro país. Sin embargo, este no es sólo un problema local, ya que, en la mayoría de los países sudamericanos, la epidemiología de la infección por Campylobacter se desconoce por completo ${ }^{22}$. de laboratorios privados ${ }^{10}$. Si comparamos con el último boletín sobre la vigilancia de Salmonella, donde en un período de cinco años (2012-2016) se remitieron 11.181 cepas al ISP, se puede comprender la dimensión de la falta de diagnóstico de la campilobacteriosis en el país, lo que conlleva una vigilancia epidemiológica ineficaz ${ }^{34}$.

Debido a esta falta de información, generalmente los escasos datos epidemiológicos de este enteropatógeno en Chile los han generado laboratorios de investigación universitarios de forma esporádica ${ }^{5}$. Es así que se han hecho intentos por comprender la relación entre las cepas aisladas de casos clínicos y de muestras de alimentos. En al año 2011, Rivera y cols. ${ }^{31}$, determinaron que los genotipos (mediante RAPD-PCR) y serotipos aviares de $C$. jejuni no fueron encontrados en cepas de origen humano en la ciudad de Concepción, lo que los autores atribuyen a que podría existir otra fuente de infección. Sin embargo, González-Hein y cols. ${ }^{32}$, demostraron que existe una relación genética estrecha (mediante PFGE) entre aislados de C. jejuni de origen humano y de origen animal, tales como carne de pollo y bovinos. Estos resultados son contradictorios y podrían estar influenciados por la cantidad de cepas analizadas, su origen y por el método de tipificación, entre otros aspectos a considerar. En un estudio más reciente, se genotipificaron 47 cepas obtenidas de casos de gastroenteritis humana (17 aisladas en la ciudad de Santiago y 30 aisladas en Valdivia) mediante tipificación por secuenciación multilocus (MLST) ${ }^{29}$. Entre los resultados más importantes, podemos mencionar que se encontró una heterogeneidad de los genotipos de ambas zonas geográficas. De hecho, sólo cinco genotipos o secuencias tipo (ST) fueron comunes, tanto a cepas de Valdivia como de Santiago; sin embargo, 14 genotipos fueron exclusivamente de Valdivia y otras siete ST fueron exclusivas de Santiago. Aunque los resultados se deben analizar con cautela, por el escaso número de cepas estudiadas, sugieren que existen algunas diferencias en la epidemiología y trasmisión de la campilobacteriosis en el centro y sur de Chile. Por otra parte, también se demostró que la mayoría de las cepas resistentes a fluoroquinolonas se agruparon en tres linajes genéticos

\section{Conclusiones y perspectivas}

En los últimos 20 años se ha logrado avanzar lentamente en la comprensión de la prevalencia y rol clínico de Campylobacter spp. en infecciones gastrointestinales y sistémicas en Chile, principalmente por esfuerzo esporádico de grupos de investigación. Sin embargo, aún estamos lejos de tener una visión más fidedigna sobre el impacto de este patógeno en la salud pública chilena. Además, son insuficientes los datos sobre resistencia antimicrobiana y su posible origen en nuestro país.

Es necesario encontrar la fórmula costo-efectiva que permita la implementación del diagnóstico de Campylobacter spp. (aislamiento, identificación y antibiograma) en los laboratorios clínicos a lo largo del país, como también se debería evitar un tratamiento antimicrobiano empírico de las gastroenteritis, con el objetivo de disminuir la resistencia antimicrobiana de este y otros enteropatógenos ${ }^{4}$.

Para mejorar la vigilancia epidemiológica, es necesario obtener las cepas de Campylobacter spp. y derivarlas al laboratorio de referencia. Sin embargo, teniendo en cuenta que la falta de implantación del diagnóstico es debido principalmente por el alto costo del cultivo, el desafío es desarrollar métodos independientes del cultivo (métodos moleculares o inmunológicos) de bajo costo, que permitan saber “a priori” cuándo una muestra está positiva para así sólo cultivar estas muestras, con el objetivo de obtener la cepa y poder utilizarla para fines epidemiológicos ${ }^{4}$.

Luego de examinar la situación de la campilobacteriosis en las últimas dos décadas en Chile y determinar cuáles son los desafíos que quedan para lograr un diagnóstico y vigilancia efectivos en el país, es de esperar que los datos aquí expuestos sean de utilidad, tanto para la toma de decisiones en políticas de salud, así como antecedentes

Agradecimientos. El autor agradece a los revisores anónimos de este manuscrito. para la formación continua de profesionales de la salud. 


\section{Referencias bibliográficas}

1.- Kaakoush N O, Castaño-Rodríguez N, Mitchell H M, Man S M. Global epidemiology of Campylobacter infection. Clin Microbiol Rev 2015; 28 (3): 687-720. doi: 10.1128/ CMR.00006-15.

2.- Newell D G, Mughini-Gras L, Kalupahana R S, Wagenaar J A. Campylobacter epidemiologysources and routes of transmission for human infection. In: Campylobacter: Features, Detection, and Prevention of Foodborne Disease 2017/Klein, Günter. Elsevier Inc. Academic Press - ISBN 9780128036235 - p. 85 - 110. https://doi.org/10.1016/B978-0-12803623-5.00005-8.

3.- O'Brien S J. The consequences of Campylobacter infection. Curr Opin Gastroenterol. 2017; 33(1): 14-20. doi: 10.1097/MOG.0000000000000329.

4.- World Health Organization. The global view of campylobacteriosis: report of an expert consultation, Utrecht, Netherlands, 9-11 July 2012. www.who.int/iris/ bitstream/10665/80751/1/9789241564601_eng. pdf

5.- World Health Organization. The increasing incidence of human campylobacteriosis report and proceedings of a WHO consultation of experts, Copenhagen, Denmark, 21-25 November 2000. https://apps.who.int/iris/ handle/10665/67767

6.- Luangtongkum T, Jeon B, Han J, Plummer P, Logue C M, Zhang Q J. Antibiotic resistance in Campylobacter: emergence, transmission and persistence. Future Microbiol 2009; 4 (2): 189 200. doi: 10.2217/17460913.4.2.189.

7.- Du Y J, Wang C Q, Ye Y L, Liu Y, Wang A M, Li Y, et al. Molecular identification of multidrug-resistant Campylobacter species from diarrheal patients and poultry meat in Shanghai, China. Front Microbiol 2018; 31(9): 1642. doi: 10.3389/fmicb.2018.01642.

8.- World Health Organization. Global priority list of antibiotic-resistant bacteria to guide research, discovery, and development of new antibiotics. 2017 http://www.who.int/ medicines/publications/global-priority-listantibiotic-resistant-bacteria/en/

9.- García P C, Valenzuela N S, Rodríguez M V, León E C, Fernández H J. Antimicrobial susceptibility of Campylobacter jejuni isolates from stool cultures in Santiago, Chile. Rev Chilena Infectol 2009; 26: 511-4. doi: /S071610182009000700004

10- Instituto de Salud Pública de Chile. Vigilancia de laboratorio de Campylobacter sp. Chile, 2005-2013. En: Boletín Instituto de Salud Pública, Vol. 4, No. 1, enero 2014. http:// www.ispch.cl/sites/default/files/Boletín\%20 Campylobacter.pdf.

11.- Braun S, Camponovo R, Cona E, Fernández
A, García P, González P, et al. Consenso. Síndrome diarreico agudo: Recomendaciones para el diagnóstico microbiológico. Rev Chil Infect 2002; 19: 101-13. http://dx.doi. org/10.4067/S0716-10182002000200006.

12.- Chanqueo C L, García C P, León C E, Blu F A. Evaluación de la tinción de Hucker para la búsqueda rutinaria de Campylobacter sp. en el estudio de un síndrome diarreico agudo. Rev Chilena Infectol. 2005; 22(3): 242-6. doi: 10.4067/s0716-10182005000300004.

13.- Procop G, Churchill D, Hall G, Janda W, Koneman E, Woods G, et al. Koneman. Diagnóstico microbiológico: Texto y Atlas. 2017. Wolters Kluwer Health.

14.- Agencia Chilena para la Inocuidad y Calidad Alimentaria. Campylobacter spp. en carne de aves de corral, Chile. Perfil de Riesgo/ ACHIPIA N01/2017. https://www.achipia. gob.cl/wp-content/uploads/2018/05/Perfil-deRiesgo-Campylobacter-spp-en-carnes-de-avesde-corral-v1-2017.pdf

15.- Porte L, Varela C, Haecker T, Morales S, Weitzel T. Impact of changing from staining to culture techniques on detection rates of Campylobacter spp. in routine stool samples in Chile. BMC Infect Dis 2016; 16: 196. doi: 10.1186/s12879-016-1546-7.

16.- Collado L, Gutiérrez M, González M, Fernández H. Assessment of the prevalence and diversity of emergent campylobacteria in human stool samples using a combination of traditional and molecular methods. Diagn Microbiol Infect Dis 2013; 75: 434-6. doi: 10.1016/j.diagmicrobio.2012.12.006.

17.- Valenzuela C, Legarraga P, Peña A, Arenas A, Berkowitz L, Ramírez G, et al. Etiologic and clinical characterization of community acquired gastroenteritis in adult patients in a Chilean emergency room by the FilmArray GI panel. PLoS One. 2018; 13(11): e0207850. doi: 10.1371/journal.pone.0207850.

18.- Prado V, Martínez J, Reyes L, Ducheylard M, Bercovich M, Millán V, et al. Intestinal Campylobacter jejuni infection in Chilean infants. Rev Med Chile. 1985; 113(6):521-5. PMID: 3834562.

19.- Levican A, Ramos-Tapia I, Briceño I, Guerra F, Mena B, Varela C, et al. Genomic analysis of Chilean strains of Campylobacter jejuni from human faeces. Biomed Res Int. 2019; 1902732. doi: 10.1155/2019/1902732.

20.- Fresia P, Jara R, Sierra R, Ferrés I, Greif $\mathrm{G}$, Iraola $\mathrm{G}$, et al. Genomic and clinical evidence uncovers the enterohepatic species Helicobacter valdiviensis as a potential human intestinal pathogen. Helicobacter 2017; 22. doi: 10.1111/hel.12425.

21.- Mardones G, López J. Implications of Campylobacter spp. as a foodborne pathogen. Chilean J. Agric. Anim. Sci. 2017; 33(1): 73-83. https://scielo.conicyt.cl/pdf/chjaasc/ v33n1/0719-3890-chjaasc-02005.pdf

22.- Fernández H. Campylobacter and campylobacteriosis: a view from South America. Rev Peru Med Exp Salud Pública. 2011; 28(1): 121-7. doi: 10.1590/s172646342011000100019.

23.- Lapierre L, Gatica M A, Riquelme V, Vergara C, Yáñez J M, San Martín B. et al. Characterization of antimicrobial susceptibility and its association with virulence genes related to adherence, invasion, and cytotoxicity in Campylobacter jejuni and Campylobacter coli isolates from animals, meat, and humans. Microb Drug Resist 2016; 22: 432-44. doi: 10.1089/mdr.2015.0055.

24.- Ministerio de Salud. Evaluación del Programa Nacional de Vigilancia Microbiológica en Alimentos, año 2015. http://www.deis.cl/wpcontent/uploads/2016/12/Evaluaci\%C3\%B3ndel-Programa-de-Vigilancia-Microbiologicaa\%C3\%B1o-2015.pdf

25.- Fernández H, Gesche W, Montefusco A, Schlatter R. Wild birds as reservoir of thermophilic enteropathogenic Campylobacter species in southern Chile. Mem Inst Oswaldo Cruz. 1996; 91(6): 699-700. doi: 10.1590/ s0074-02761996000600007.

26.- Ochoa S, Barrientos C, Ojeda J, Collado L. Evaluación del rol del perro doméstico como potencial reservorio de especies patógenas emergentes de los géneros Campylobacter y Helicobacter. Rev Chilena Infectol 2017; 34 (Supl 1): CO 8, pág. S75

27.- Levican A, Flores O, Sánchez S, Bascuñán M, López R, Ojeda K, et al. Faecal shedding of campylobacteria among domestic and wild animals from an urban coastal area. Austral J. Vet. Sci. 2019; 51(2): 83-90. doi.org/10.4067/ S0719-81322019000200083.

28.- Fernández H, Mansilla M, González V. Antimicrobial susceptibility of Campylobacter jejuni subsp. jejuni assessed by E-test and double dilution agar method in Southern Chile. Mem Inst Oswaldo Cruz 2000; 95: 247-9. DOI: 10.1590/s0074-02762000000200020.

29.- Collado L, Muñoz N, Porte L, Ochoa S, Varela C, Muñoz I. Genetic diversity and clonal characteristics of ciprofloxacinresistant Campylobacter jejuni isolated from Chilean patients with gastroenteritis. Infect Genet Evol 2018; 58: 290-3. doi: 10.1016/j. meegid.2017.12.026.

30.- Millanao A R, Barrientos-Schaffeld C, SiegelTike C D, Tomova A, Ivanova L, Godfrey HP, et al. Antimicrobial resistance in Chile and The One Health paradigm: Dealing with threats to human and veterinary health resulting from antimicrobial use in salmon aquaculture and the clinic. Rev Chilena Infectol. 2018; 35(3):299308. doi: 10.4067/s0716-10182018000300299.

31.- Rivera F N, Bustos B R, Montenegro H S, Sandoval M M, Castillo N J, Fernández J H, 
et al. Genotyping and antibacterial resistance of Campylobacter spp strains isolated in children and in free range poultry. Rev Chilena Infectol 2011; 28:555-62. doi: /S071610182011000700008 .

32.- González-Hein G, García P, Foerster C, Troncoso M, Figueroa G. Campylobacter jejuni isolated from human cases in Chile showed indistinguishable PFGE profiles with strains isolated from poultry and bovine sources. Cyta J Food. 2013; 11: 185-9. https://doi.org/10.108 0/19476337.2012.722564.

33.- Vidal-Veuthey B. Análisis molecular de la resistencia antibiótica en cepas clínicas de Campylobacter jejuni aisladas en Chile. 2019. Tesis del Programa de Magister en Ciencias mención Microbiología. Universidad Austral de Chile.

34.- Instituto de Salud Pública de Chile. Boletín de Vigilancia Epidemiológica. Salmonella spp. 2012 - 2016, Vol. 6, No. 9, septiembre 2016. http://www.ispch.cl/sites/default/files/ BoletinSalmonella-23012017A.pdf

35.- Durán C, Chandía S, Arellano C, Benadoff D, Zamorano A, Silva F, et al. Evaluación de método inmunocromatográfico Immunocard stat! Campy para el diagnóstico de Campylobacter jejuni/coli en muestras de deposición, obtenidas de niños con diarrea. Rev Chilena Infectol. 2011; 28 (Supl 2): P 27, pág. S73.

36.- Fuenzalida L M, Camponovo R, Domínguez
M, Ulloa M T. Evaluación de la técnica inmunocromatográfica Certest Campylobacter (Biotec $\left.{ }^{\circledR}\right)$ para el diagnóstico rápido de Campylobacter spp. en deposiciones. 2014. XXXI Congreso Chileno de Infectología, Puerto Varas, Chile. 13-15 de noviembre de 2014. Pág 160, P89. http://www. sochinf.cl/portal/templates/sochinf2008/ documentos/2014/Libro_congreso_2014.pdf

37- González-Hein G, Cordero N, García P, Figueroa G. Molecular analysis of fluoroquinolones and macrolides resistance in Campylobacter jejuni isolates from humans, bovine and chicken meat. Rev Chilena Infectol. 201330 (2):135-9. doi: 10.4067/S071610182013000200003 